\title{
Enthymematic Reasoning and Pragmatics
}

\subsection{Introduction}

There is an obvious connection between on the one hand enthymematic reasoning and topoi, and on the other pragmatic phenomena like implicature, presupposition and different types of discourse relations. In this chapter we will briefly discuss the relation between rhetorical reasoning drawing on topoi and such phenomena.

First, in Section 2.2 we will look at presupposition (Strawson, 1950; Stalnaker, 1974; Karttunen, 1974), moving on to Grice's account of conversational implicature (Grice, 1975) in Section 2.3. In Sections 2.4 and 2.5 we are still concerned with inference, considering the approach to implicature taken in Relevance Theory (Sperber and Wilson, 1995), and the anti-inferentialist view of inference presented in Recanati (2004). In Section 2.6 we will consider sense-making of longer strands of discourse and how we assign rhetorical relations between sentences or utterances.

We do not claim that this account of topics related to enthymemes and topoi is exhaustive. It would probably be possible to fill a book with a relevant discussion on the relation between enthymemes and implicature alone. However, we hope that this chapter will give an overview and answer some questions, while also raising many new ones.

\subsection{Presupposition}

The classic, semantic, definition of presupposition is that it is an inference which survives embedding under negation (see for example Strawson, 1950). Stalnaker (1974) argued against the notion of semantic presupposition and diagnostics such as the negation test, in favour of a pragmatic analysis. In (2.1a) and (2.1b) we see Stalnaker's definition of semantic and pragmatic presupposition respectively.

(2.1) a. ... a proposition that $P$ presupposes that $Q$ iff $Q$ must be true in order that $P$ have a truth-value at all (Stalnaker, 1974 p. 48).

b. ... something like the background beliefs of the speaker-propositions whose truth he takes for granted, or seems to take for granted, in making his statement (Stalnaker, 1974 p. 48). 
An example of an utterance that would carry a presupposition according to the definition in (2.1a) is (2.2), which presupposes that there is a queen of England; this needs to be true for the sentence to be true or false.

(2.2) A: The queen of England is bald.

The presupposition of (2.2) would be the same regardless of which of the definitions in (2.1) we use. However, the pragmatic definition would include other types of inferences too, which would not fall under presupposition according to the semantic definition in (2.1b). Let us consider one of the examples from Chapter 1, repeated in (2.3):

(2.3) The bride is pregnant, so I might drop by in the wee hours!

The example in (2.3) conveys the enthymeme in (2.4).

$$
\frac{\text { the bride is pregnant }}{\text { I might drop by in the wee hours }}
$$

According to Stalnaker's definition in (2.1b), (2.4) carries a (pragmatic) presupposition that a pregnant bride is a reason for a wedding party to end early. This indicates that topoi sometimes are pragmatic presuppositions. However, this is not very specific, since pragmatic presupposition seems to encompass a whole array of ways of communicating implicit meaning. The example in (2.4), for example, is similar to that given by Grice (1975) to illustrate the notion of conventional implicature:

(2.5) a. S: He is an Englishman. He is, therefore, brave (Grice, 1975).

b. Conventional implicature: If someone is an Englishman, he is brave.

Grice claims that the word therefore in (2.5a) gives rise to the conventional implicature in $(2.5 \mathrm{~b})$. From a micro-rhetorical point of view, we can see $(2.5 \mathrm{a})$ as an enthymematic argument that a particular person will be brave since he is an Englishman, based on a topos that if someone is an Englishman then that someone will be brave.

So, we have established that topoi may function as pragmatic presuppositions, and that some topoi could also be categorised as conventional implicatures. However, a topos cannot be the exact same thing as a conventional implicature, since some enthymemes lack a lexical item that conventionally implicates a particular structure between its constituents. Let us consider, 
for example, (2.6), an authentic dialogue example originally used by Walker (1996). The turn in the excerpt consists of two utterances produced by the same speaker $A$ - the speaker - and $B$ - the addressee - are two colleagues on their way to work. They meet up somewhere along the way and continue their walk together. This is something they often do, and they are thus both familiar with the surroundings as well as with the physical goal of the walk.

(2.6) A: Let's walk along Walnut Street. It's shorter.

For this turn to make sense to an addressee, they would have to assume that the speaker presupposes some kind of link between Walnut Street being shorter and the suggestion to walk along Walnut Street. However, since there is no specific word in (2.6) which warrants this assumption, this link cannot be a conventional implicature. Instead it seems to be the case that the rhetorical structure of (2.6) — and thereby part of its meaning — depends on the dialogue participants already having access to a notion that a route being short is a reason for choosing that route. In micro-rhetorical terms we would say that this notion is a topos, which may also be a subtype of a more abstract topos having to do with convenience, efficiency, etc.

\subsubsection{Accommodation}

The process of adding a presupposition (semantic or pragmatic) to the discourse model is usually referred to as accommodation. This phenomenon was discussed by Stalnaker (1974) and Karttunen (1974), but the term was coined by Lewis (1979).

Lewis illustrates the notion of presupposition accommodation with the example (2.7), where (2.7a) is ok but (2.7b) seems odd.

(2.7) a. Fred has children, all Fred's children are asleep.

b. All Fred's children are asleep, and Fred has children

The reason for this, argues Lewis, is that the proposition All Fred's children are asleep presupposes that Fred has children. Thus, the belief that Fred has children is already integrated in the discourse model for speaker and addressee alike, and adding it a second time is redundant.

1 The term utterance is sometimes defined as a string of words produced by a speaker while said speaker is holding the floor. We will use the term turn to refer to such a unit, and reserve utterance for units that convey an atomic proposition, something like what Schlangen (2005) refers to as intentional units. 
The presupposition in (2.7) is a semantic presupposition triggered by the possessive Fred's children, but Lewis' definition of presupposition accommodation in (2.8) seems to include any type of pragmatic presupposition.

(2.8) If at time $t$ something is said that requires a presupposition $P$ to be acceptable, and if $P$ is not presupposed just before $t$, then-ceteris paribus and within certain limits-presupposition $P$ comes into existence at $t$ (Lewis, 1979 p. 340).

This means, for example, that if nobody in the discourse context objects when a speaker utters (2.6), the assumption of a link between the route being short and the advantage of choosing it will be accommodated in the conversation. However, if someone says something like "What do you mean shorter-why would we want to choose the shortest route?", this is evidence that the pragmatic presupposition - or topos - has not been accommodated in that speaker's discourse model. Stalnaker (1998) points out that in order for accommodation to work a speaker cannot expect controversial assumptions to be accommodated, and quotes Heim (1992):

One may explicitly assert controversial and surprising things (in fact one should) but to expect one's audience to accept them by way of accommodation is not good conversational practice.

In rhetorical terms we could say that it is not wise to choose an enthymeme which is not already associated with a suitable topos that the addressee has access to, or that evokes a topos which is acceptable to the audience.

Lewis shows that the principle of accommodation applies to several linguistic phenomena, such as definiteness, modal expressions, etc. To that list we would like to add topoi — when a topos which is necessary for an enthymematic argument to make sense is added to the discourse model — and enthymemeswhen a topos in the resources of an agent causes her to assign an enthymematic structure to the discourse.

In this section we have shown that topoi are included in Stalnaker's wide definition of pragmatic presupposition. In addition to this, in some cases topoi are to be considered conventional implicatures, in the sense of Grice (1975). Whether a topos is conventionally implicated or not depends on whether the enthymematic structure is established by means of lexical items signaling that the speaker assumes a causal relation between the premise and conclusion of the enthymeme.

There is a distinctly rhetorical dimension to accommodation of presuppositions as well as accommodation of other phenomena, in that it may allow 
content to be smuggled into the discourse and trick hearers into accepting-or at least not objecting to- things they would reject if they were said explicitly. We suggest that we could talk about accommodation of both topoi (as types of pragmatic presuppositions) and enthymemes (argumentative structure). We also suggest that the notion of "surprising" or "controversial" presuppositions which Stalnaker (1998) and Heim (1992) argue should not be left to accommodation according to good conversational practices, are presuppositions which cannot be identified as belonging to a topos that is acceptable by the agent in question.

\subsection{Conversational Implicature}

Grice's theory of conversational implicature is an attempt to systematically describe how it is possible for language users to convey (and mean) moreor something different-than the truth-conditional content of an utterance. Grice (1975) distinguishes between what is said and what is implicated. What is said corresponds to the truth-conditional meaning of an utterance and what is implicated to what a speaker conveys by uttering a certain string of words in a certain context, assuming (though perhaps not aware of) the principle of cooperation elaborated as four maxims of rational and efficient communication. In the exchange in (2.9) below, from Grice (1975), $B$ 's reply that there is a garage around the corner would not be very helpful if $B$ knew the garage to be closed, not to sell petrol, etc.
$(2.9)$
a. $A$ : I am out of petrol
b. $B$ : There is a garage around the corner

$A$ expects $B$ 's utterance to be a relevant, truthful and complete reply, based on background knowledge and an assumption that $B$ is being cooperative. As mentioned, Grice specifies the assumptions of the Cooperative Principle further in the four maxims of conversation (paraphrased):

1 Quantity: Make your contribution informative enough, but do not say more than is required.

2 Quality: Be truthful, or at least only say what you believe to be true.

3 Relation: Be relevant.

4 Manner: Be orderly, avoid ambiguity, etc.

By adhering to, or blatantly ignoring ( flouting) the maxims and the cooperative principle, a speaker may express a lot more than the truth-conditional content 
of his/her utterance. So, Grice would say that we would interpret (2.9b) as implicating that the garage is open (or at least that the speaker believes this) due to the maxim of relation, since the information that there is a garage around the corner would otherwise be irrelevant. Let us now consider (2.10), which we looked at in the previous section. In Gricean terms it could be analysed like this:

(2.10) a. $A$ : Let's walk along Walnut Street.

b. $A$ : It's shorter.

c. Implicature: Walnut Street being shorter is a good reason for choosing Walnut Street.

Due to the maxim of relation, we as language users want to interpret (2.10b) as a relevant contribution. One way of doing this would be to interpret it as a relevant reason for choosing Walnut Street. However, it seems to us that we need something more than the Cooperative Principle and the maxims to get at the correct implicature in (2.10). We can illustrate this by comparing (2.10) with the similar dialogue (2.11):
(2.11) a. A: Let's walk along Walnut Street.
b. A: It's longer.
c. Implicature: ??

Now, by simply applying the cooperative principle and the maxims $A$ 's conversational partner $B$ might be able to infer that $(2.11 b)$ is relevant in relation to (2.11a). However, if $B$ 's resources do not include a topos underpinning (2.11b) as a premise in an argument for choosing Walnut Street - such as longer routes are better or it is preferable to spend longer time doing things - it would be hard for $B$ to arrive at a relevant interpretation. This is of course context dependentthere are some things that we like spending as long time as possible doing, and if walking is one of them, it is natural that a longer route is preferred. If $B$ knew that $A$ is always interested in getting exercise, which of course a longer route would provide in greater measure, $B$ might also be able to derive the relevant interpretation of (2.11). However, considering the notions most of us have about comfort, efficiency, etc. (2.10) seems like less of a stretch unless the context is set up in a specific way. So, even if the maxims tell us that we should try to interpret contributions as relevant, true, etc., in order to do this we need some underpinning in the form of a pattern of reasoning - a topos - that fits in with the contribution we are trying to make sense of. If we consider yet another manipulation of our original example, this is even more clear: 
(2.12) a. A: Let's walk along Walnut Street.

b. $A$ : In the European Union, labour market conditions showed no signs of improvement during 2013.

The contribution in $(2.12 \mathrm{~b})$ is very difficult to make sense of in the context of (2.12a), and would probably be taken as relating to an earlier utterance or as $A$ abruptly changing the subject (however, it cannot be excluded that it could be interpreted as relevant under particular circumstances).

So, even though it appears that we use some notions corresponding to Grice's principle of cooperation and related maxims to interpet enthymemes, we need access to some underpinning pattern or topos to actually arrive at an interpretation. If we do not have access to relevant topoi, or if the contribution does not contain enough information to point us in the direction of a relevant topos, we have difficulty making a relevant interpretation. In cases like these we may get additional information by making a clarification request. Imagine for example a context of (2.9) where $B$ would be totally unaware that you can buy petrol in a garage. The dialogue in (2.9) could then play out as in (2.13):
(2.13) a. $A$ : I am out of petrol
b. $B$ : There is a garage around the corner
c. A: What do you mean garage-I need to buy petrol?
d. $B$ : They sell petrol
e. $A$ : Ah-ok!

To conclude, it seems that the principles suggested by Grice lead us in some cases to infer that a speaker means something more than what is said. However, if we have access to relevant topoi we can understand not only that something is implicated but what is implicated. If we, on the contrary, do not have access to a topos that fits the discourse, it is difficult for us to make sense of an utterance where part of the meaning is conversationally implicated. Some conversational implicatures can be accounted for by other means, for example scalar implicatures (Horn, 1984). However, in the case of relevance implicature for example, we need something like a topos to produce and interpret contributions.

\section{$2.4 \quad$ Relevance Theory}

While the neo-Gricean developments of implicature theory have reduced the maxims to a smaller set of communicative principles, Relevance Theory (Sperber and Wilson, 1995; Wilson and Sperber, 2004; Carston, 2006) has replaced 
them with just one principle of relevance. Unlike the maxims, this principle is not perceived as a communicative norm based on assumptions of cooperation and rationality, but rather as a fundamental feature of human cognition.

Unlike Gricean pragmatics, which distinguishes between what is said - the truth-conditional content of an expression-and what is implicated, Relevance theory distinguishes between explicature and implicature, where explicature refers to the explicit content of an utterance in a particular context. For example, we would generally interpret (2.14a) as Jack and Jill being married to each other rather than to other people. According to Relevance theory this is the explicit, truth-evaluable content - the explicture-of the utterance, which is arrived at through disambiguation, reference resolution and other pragmatic processes.

\section{(2.14) a. Utterance: Jack and Jill are married \\ b. Explicature: Jack and Jill are married to each other.}

Meaning interpretation in Relevance Theory is not perceived as something that happens in steps where one step is executed after the other (primary and secondary processes). Instead, the interpretation process is seen as incremental, and hypotheses about explicatures, implicated premises and implicated conclusions are developed in parallel. According to Sperber and Wilson (2004) this is an inferential process, i.e. if certain explicatures are assumed, certain implicated premises (intended contextual assumptions) and implicated conclusions (implicatures) follow by necessity. This is illustrated very well by the example in (2.15) (Sperber and Wilson, 1995):
(2.15) a. Peter: Would you drive a Saab?
b. Mary: I wouldn't drive ANY Swedish car.
c. Implicated premise: A Saab is a Swedish car
d. Implicated conclusion: Mary wouldn't drive a Saab

In (2.15) the implicated conclusion (implicature) is that Mary would not drive a Saab, based on the implicated premise that a Saab is a Swedish car. According to Relevance Theory, this premise presents itself automatically due to the relevance assumption - if a Saab were not a Swedish car, why would Mary answer the way she did? However, if Peter thought that a Saab is a German car, the implicated premise and conclusion might not be obvious to him, despite the relevance assumption. And if a Saab were not, in fact, a Swedish car, and Peter knew this, the relevance assumption alone would probably not be enough for the interaction to run smoothly. 
The example in (2.15) is neat, since the relevance assumption seems to do the work unless one of the agents involved in the dialogue lacks necessary background knowledge. However, in (2.16), an example taken from Carston and Hall (2012), the situation is not as straightforward:
(2.16) a. Max: How was the party?
b. Amy: There wasn't enough to drink and everybody left early

Here we would probably infer the implicated conclusion that the party was not a success, based on implicated premises that "people leaving early" and "lack of drink" are associated with "dull party". As previously discussed, given the implicated premise in (2.15), the implicated conclusion follows by necessity. However, in (2.16), this is not the case. For the implicated premises to necessarily lead to the conclusion that the party was unsuccessful, they would have to be universal rules. For example, the implicated premise "every party at which there is not enough drink is unsuccessful" would, in any context, lead to the conclusion that a particular party at which there was not enough drink was unsuccessful. However, this seems unsatisfactory. Many would agree that a low drink supply is acceptable as an explanation for why a party is unsuccessful, but there might also be exceptions to this.

\subsubsection{Relevance, Enthymemes and Topoi}

Like Gricean and neo-Gricean theories of implicature, Relevance Theory provides an account for why we interpret utterances like (2.16b) as conveying more than the truth-conditional content. However, both Gricean and Relevance Theoretical accounts require some underpinning for the implicated inferences. The theory that we propose accounts for the nature of the implicated premises, and also for how implicated conclusions are generated. For example, let us consider (2.16) in terms of enthymematic reasoning.

(2.17) there wasn't enough drink at the party and everyone left early the party was not a success

Rather than viewing this enthymeme as an incomplete argument requiring a set of implicated premises which necessarily lead to the implicated conclusion, we suggest that the enthymeme is underpinned by more general topoi, licensing the proposition of the antecedent as an acceptable reason for the proposition of the conclusion. The topos that parties at which there is too little to drink are unsuccessful does not hold in all cases, but, if we agree with it, we would probably also agree that it is more acceptable the more it fits in with the 
situation at hand. That is, if there is almost nothing to drink at a party, it is very likely for the party to be unsuccessful. This is a good example of what Ducrot (1988) refers to as the graduality of topoi (see Section 1.4.1).

The second topos invoked here is one linking "bad party" to "guests leaving early". This might not be a rule that specifically concerns parties and guests, but rather one whose gist is that if people do not like something, they tend to leave-people vote with their feet. We agree with the view that there are implicit premises in dialogues such as (2.16), and that these to some degree have to be anticipated by the speaker and accommodated by the listener. However, we believe that these implicit premises are not precise, but rather an instantiation in the dialogue situation of one or more general topoi.

\subsection{Anti-inferentialism}

Unlike the Relevance Theoretical view, the anti-inferentialist (Recanati, 2001, 2004) view of pragmatic meaning in context attributes many aspects of utterance meaning to non-inferential processes. On this view interpretation of an utterance happens in two steps-via primary pragmatic processes and secondary pragmatic processes, where the primary processes correspond roughly to Relevance Theory's concept of explicature. However, the distinguishing characteristic of primary pragmatic processes is that they do not require the prior identification of some proposition. They are not conscious, since a "normal" language user is unaware of the processes through which the context-free meaning is enriched to fit the situation. Primary pragmatic processes may be bottom up, i.e. they are linguistically mandated, or top down, i.e. they are contextually driven. Recanati (2004) lists four different types of primary pragmatic processes:

- Saturation: Mandatory, linguistically motivated disambiguation e.g. anaphor resolution. (Bottom up)

- Free enrichment: The sentence She took out the key and opened the door would generally be interpreted in a way such that the key that was taken out was the key used to open the door. Usually, free enrichment corresponds to the specification of some expression in the utterance by making it contextually more specific.

- Loosening:When a concept is used more generally than the literal interpretation allows, for example, in the utterance the ATM swallowed my credit card, the aspects of swallowing that the speaker refers to are more general than what we connect with actual swallowing by a living creature.

- Semantic transfer: In the utterance The ham-sandwich left without paying, the ham-sandwich refers to the person who ordered the ham-sandwich- 
not to the dish itself. I'm parked out back does not mean that the person who performs the utterance is parked out back but that his or her car (or other vehicle) is.

The anti-inferentialist take on interpretation is characterised by the belief that all pragmatic processes which are necessary to arrive at a truth conditional interpretation are perceived as being non-inferential. Of the different types of primary processes mentioned above, all except saturation are contextually mandated (top-down), and these are the ones that interest us most. Recanati proposes that "interpretation is as direct as perception" in these cases, and instead of looking to logic to explain for example enrichment, he turns to association between suitable schemata (Rumelhart, 1980) or frames (Fillmore, 1982) and context free utterance meaning in order to explain how we interpret contextual meaning.

The concept of schema has been around in cognitive science since the seventies at least, and can be described as a system for organising knowledge (or beliefs). Recanati (2004) sketches a picture of how schemata play a role in interpretation: An expression activates a cognitive schema, which is basically a connection between two (or more) semantic values that says that these values fit together. As an example, Recanati considers (2.18).

\section{(2.18) John was arrested. He had stolen a wallet}

Recanati attributes the reference resolution in (2.18) to the fact that (most of us) have access to a schema where "stealing" and "being arrested" are linked. Rumelhart (1980) describes the internal structure of a schema as the script of a play where actors who can fill the different roles of a play in different renditions correspond to variables which can be associated with different aspects of the schema on different instantiations.

\subsubsection{A Rhetorical View of Anti-inferentialism}

We consider (2.18) as an enthymematic argument where the conclusion is that John was arrested, and the premise that he stole a wallet, as seen in (2.19).

(2.19) $\frac{\text { John had stolen a wallet }}{\text { John was arrested }}$

This enthymeme is underpinned by a topos linking stealing to getting arrested, as seen in $(2.20)$.

(2.20) If someone steals something, they get arrested 
The topos in (2.20) is a warrant for the enthymeme in (2.19), since (2.19) is easily recognisable as an instantiation of $(2.20)$.

In many ways a theory of topoi such as the one in (2.20) is compatible with the anti-inferentialist view that knowledge and beliefs are organised in terms of schemata and frames, making up the cognitive resources available to a language user, based on the experiences of that individual. Like the theory we propose, anti-inferentialism emphasises the importance of context and cognitive resources for meaning interpretation. However, topoi-as they are traditionally perceived in rhetoric - tend to be less domain specific than schemata. This means that one topos could be relevant to one particular association within a schema but irrelevant to-or even inconsistent with—another association within the same schema. Also, one topos may be relevant to, or fit into, several schemata in different domains.

Recanati makes a point of primary pragmatic processes being associative rather than inferential. He defines these processes as being as automatic as perception and non-conscious for the "normal" language user engaged in interaction, while inference to Recanati is something that the normal language user consciously reasons about. However, there seems to be some evidence that a process which is conscious for an individual at one point in time can be nonconscious at some later point in time. For example, Swedish speaking students of French may at first have to think carefully every time they choose which form of a verb to use, due to the fact that Swedish verbs, unlike French, are not conjugated by person. After a while this process will be quicker and less conscious, and eventually, as the students learn to master the language, it becomes more or less automatic.

Dreyfus and Dreyfus (1980) describe the acquisition of skills in terms of five stages, where stage five (highly competent) involves a lot of tacit knowledge and routinised behaviour, while stage one (beginner) involves almost exclusively conscious reasoning. From this view point, if it were true that all enrichment which is necessary to reach the contextualised, fully enriched, truthconditional content of any sentence, were associative (automatic) it would be difficult to explain language learning.

It would be possible to argue that anti-inferentialist theory is not about language learning, it is an account of the interpretation processes of a fully competent speaker of a language, so this objection is not relevant. However, we would like to argue that even a fully competent language user continues to incorporate new interpretations of expressions and is frequently faced with new types of contexts in which old (and new) expressions are to be interpreted-language change does not only happen between generations-it happens continuously, and the language of an individual develops and changes during the course of 
the individual's life. While we remain agnostic regarding the cognitive processes underlying different types of pragmatic interpretation, we want to be able to account for associative as well as inferential reasoning. Both convey enthymematic structures in discourse, and both must be warranted by something like topoi.

\subsection{Discourse Coherence}

We are interested in pieces of discourse where two propositions are related in the sense that they convey an enthymematic argument underpinned by a topos or a set of topoi. We argue that this type of relation contributes to coherence. In this section, we look at enthymemes and topoi in the context of coherence in dialogue and rhetorical relations.

Before we look at specific theories accounting for coherence, we will say something about the phenomenon as such. Generally, coherence is taken to mean how a discourse, text or conversation, "sticks together", and coherent refers to the property of being interpreted as belonging to the same unit. Leth (2011) suggests that the minimal requirement for coherence is relation. This means that when a number of discourse units are put in the same context, or considered in the same context, relations between these units are automatically generated in the minds of the agents involved.

On a general level, the reason for this would be that we try to relate utterances or other linguistic units to each other in some meaningful way as soon as we encounter them. Thereby we also assign a possible discourse relation between these units. The view that our cognition is constantly seeking to create coherence, and that virtually any two constituents in discourse could be interpreted as coherent under the right circumstances, seems reasonable and is supported by experimental evidence showing that dialogue participants tend to interpret non-authentic insertions in online dialogue as meaningful contributions (Healey et al., 2018). However, this does not tell us how an agent creates coherence between linguistic units, nor does it predict to what degree a linguistic contribution would be seen as relevant in a particular context. In the next two subsections, we will briefly consider two approaches to coherence, which in different ways try to elucidate these problems.

\subsubsection{Conversation Analysis}

In Conversation Analysis (CA, Sacks et al., 1974), organisation of discourse is defined in terms of adjacency pairs - pairs of functionally related dialogue contributions by two different speakers—and sequences. Each sequence revolves 
around a base adjacency pair conveying the basic action performed in the sequence. A minimal sequence consists of one adjacency pair, for example a greeting followed by another greeting by an interlocutor. However, sequences are often longer than one adjacency pair. By means of the example below, Schegloff (2007) illustrates how a sequence may be expanded with one or more inserted sequences. The context of the dialogue is a girl asking her boyfriend if she can borrow a gun for an assignment in drama class. The first part of the base adjacency pair is $B$ 's utterance in lines 9-10, which is not resolved until $J$ utters the second part of the base adjacency pair in line 93 .

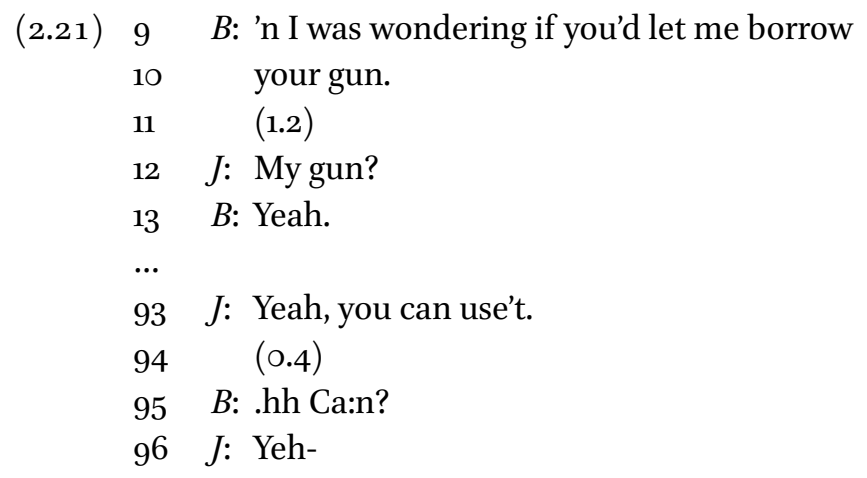

In between these turns at talk, there are several sequence expansions (CouperKuhlen and Selting, 2017) consisting of adjacency pairs. Some of these expansions are themselves nested. In addition to the functional relation within adjacency pairs, such as question-answer, request-acceptance, etc., topic is also seen as contributing to coherence. Typically, the topic does not shift between the first and second part of a base adjacency pair, and conversely, there is often a topic shift between sequences.

\subsubsection{A Micro Rhetorical Perspective on CA}

Even though there is often correspondence between the beginning and end of sequences and topic shift in conversation, there are cases where the connection is less clear (Schegloff, 2007). For example, the topic of an adjacency pair within a sequence expansion might not be the same as the overall topic of the sequence, that is, of the base adjacency pair. Take for example (2.22), an exchange within the long sequence expansion between line 9-10 and 93 in (2.21):

(2.22) $82 \quad J$ : You a good- (.) uh::: (1.8) a- actress?

$83 \quad(1.0)$ 
84 B: No: heheheh?

85 (0.5)

86 J: Th'n how d'ju come out to be A:nnie.

It is not obvious how the topic of (2.22) is related to the overall topic of (2.21), the base adjacency pair. This is true of many sequence expansions. There is often a topical link to the previous adjacency pair, but it is not always obvious. In some cases, in order to identify an utterance as the second part of an adjacency pair or the first part of a sequence expansion, rather than as an actual change of topic, a topos is required warranting the interpretation. In (2.22) the question whether $B$ is a good actress or not is on topic in virtue of a topos connecting playing the leading role and being a good actor. However, for this adjacency pair to be on topic in relation to the base adjacency pair, more topoi are required.

Even in cases where two turns at talk are clearly related, they often involve the assumption of topoi either to be present in the minds of the interlocutors, or be possible to infer from the context. Due to the ethnomethodological approach to interaction taken by $\mathrm{CA}$, which discourages assumptions of things that are not explicit in the discourse, this is to a great extent ignored in CA. One example where a topos is needed to explain coherence, is the excerpt in (2.22). The functional coherence between lines 82 and 84 is obvious. However, the topical connection to the rest of the dialogue is not clear until we see the follow-up question in line 86 . The subsection in (2.22) conveys an enthymeme like the one in (2.23):

$\underset{\text { it is surprising that } B \text { is playing Annie }}{B \text { is not a good actress }}$

This enthymeme is underpinned by a topos saying that if someone plays the lead, they are a good actor. Without this assumption, the relevance of the first part of the adjacency pair in line 82, to the first part of the subsequent adjacency pair in line 86 , is not clear. Most of the time, the topoi underpinning a dialogue, by warranting its enthymemes, are so obvious to a conversational participant that they do not require conscious reasoning. However, they still contribute to coherence. Adding enthymemes and topoi provides a more general theory of coherence in dialogue than one only drawing on adjacency pairs and sequence organisation.

\subsubsection{Rhetorical Relations}

Work within CA offers many insights to how individual utterances in dialogue relate to each other, thereby contributing to coherence. However, there are 
few attempts at generalising these insights to a theory of coherence that could be used to make predictions regarding interpretation and production of language.

CA is typically devoted to conversation, and is not concerned with coherence in other types of discourse. In contrast, theories of rhetorical relations aim at a generalisable theory of coherence which applies to many types of discourse, and is based on content rather than turn taking patterns.

We are particularly interested in Segmented Discourse Representation Theory (sDRT, Asher and Lascarides, 2003), since it-in comparison to e.g. Rhetorical Structure Theory (RST, Mann and Thompson, 1986, 1988) — does not only focus on written text but also considers dialogue data.

A basic premise of SDRT is that rhetorical relations between sentences or utterances are sometimes necessary in addition to compositional or dynamic semantics to fully interpret discourse. According to Asher and Lascarides (2003), traditional dynamic semantics (Kamp and Reyle, 1993; Groenendijk and Stokhof, 1991) —although capable of handling many coherence phenomenadoes not adequately handle rhetorical relations, nor the phenomena accounted for by rhetorical relations alone. Some of these phenomena are bridging inference, lexical ambiguity and conversational implicature. In this section we will look at some of the rhetorical relations postulated in SDRT, and consider how they relate to enthymemes and topoi.

The different types of rhetorical relations or discourse relations in SDRT are not based on rhetorical—or functional—criteria alone, but on a combination of rhetorical quality and other properties like tense, mood and discourse order. Apart from the division into main rhetorical relation typessuch as elaboration, narration and explanation - there is also a separate division of relation types into content-level relations, text-structuring relations, cognitive-level relations, divergent relations, and meta-talk relations. Some relation types have subtypes, and these subtypes may also belong to different groups. However, this fine-grained division is not relevant to us at this point, and we will settle for distinguishing between relatively coarse-grained categories. Above all, we are interested in the two types of relation which are most easily associated with enthymemes-result and its counterpart explanation.

In (2.24) we see an example given by Asher and Lascarides (2003, p. 463), where two utterances are linked by the rhetorical relation result.

(2.24) a. John pushed Max.
b. He fell. 
The relation between (2.24a) and (2.24b) is based on a notion of causation, and the order of discourse matches the temporal order in which the events take place. In (2.25) (Asher and Lascarides, 2003, p. 463) the situation is reversed. As in (2.24), the reasoning relies on a notion of causation. However, in this case the order of events does not match the discourse order, and the relation between $(2.25 \mathrm{a})$ and $(2.25 \mathrm{~b})$ is thus explanation, not result.
(2.25) a. Max fell.
b. John pushed him.

The underlying reasoning in the two cases is similarly warranted, and the difference between the relations is the order of the constituents. This is reflected in the logical forms of the axioms postulated in SDRT as underpinning the interpretations of the rhetorical relation result between the constituents of (2.24), and explanation between those of (2.25).

In (2.26), the underlying reasoning is different from that warranting (2.24) and (2.25).
(2.26) a. Max fell.
b. John helped him up.

The discourse order and temporal order match, and (2.26a) and (2.26b) are clearly related in the sense that helping someone up is something you might do if they have fallen, but Max falling is not causing John to help him up, it is merely a reason for Max being in need of being helped. This discourse is an example of the rhetorical relation narration, which relates two propositions only if the event described by the first proposition temporally precedes that of the second (see also (2.29), below).

As language users, our intuition about (2.26) is that that Max's falling precedes John's helping him up. In the case of (2.25), on the other hand, the intuition is that the falling happens after, and is caused by, the pushing.

Asher and Lascarides argue that rhetorical relations are necessary to capture the disctinct temporal content of (2.26a) and (2.26b), and that these relations are not derived from domain knowledge alone. They say, with regard to the example in (2.26):

If "pushings typically cause fallings" were part of domain knowledge, one might use it to construct the right logical form, but this proposition seems quite implausible and hence not part of domain knowledge.

ASHER and LASCARIDES, 2003 p. 7 
Instead, Asher and Lascarides suggest that we infer a causal link in virtue of the presence of a rhetorical link between the two propositions. This raises a few questions: Firstly, is it really implausible that some notion that pushings cause, typically cause, or may cause fallings, is part of domain knowledge? Secondly, how do we as agents interpreting $(2.25 \mathrm{~b})$ know that we are supposed to infer a causal link and not, for example, a narrative link?

\subsubsection{Enthymemes, Topoi and Rhetorical Relations}

With regard to (2.24) and (2.25), we may say that while the classification of the discourse relation as either result or explanation depends on the order of the sentences, the two examples convey the same enthymeme. If we accept the relations between the sentences in (2.24) and (2.25) as result and explanation respectively, we also have to accept that it was John who caused Max to fall by pushing him. We can see this discourse as expressing an enthymematic argument where Max's falling is the conclusion and the pushing of him the premise, as in (2.27).

\section{(2.27) $\frac{\operatorname{push}(\mathrm{j}, \mathrm{m})}{\text { fall }(\mathrm{m})}$}

In order for a proposition to work as an explanation, there there must be an acceptable warrant. In the case of (2.27), we have access to some cognitive resources concerning pushings and fallings, among them a rule of thumb saying that a common/possible/potential relation between pushings and fallings is that pushings cause fallings.

Now, this is not necessarily a rule based on probabilistic reasoning about the real world, but rather a salient notion that speakers may relate to the activity of pushing. Basically, it says that when falling occurs in the context of pushing, we are licensed to assume that the pushing caused the falling. We would say that the discourse in (2.27) evokes a topos like the one in (2.28):

(2.28) $\frac{\operatorname{push}(\mathrm{x}, \mathrm{y})}{\text { fall }(\mathrm{y})}$

This topos underpins the enthymeme in (2.27), and helps us accommodate the appropriate discourse relation.

We argue that some discourse relations are clearly related to enthymemes, as they contain some kind of claim or conclusion, as well as a constituent which serves as support for that claim or conclusion. But what about those relations that lack a causal link between utterances - are they unrelated to enthymemes and topoi? Consider the discourse in (2.29) Asher and Lascarides (2003, p. 462): 
(2.29) a. Max came into the room

b. He sat down

c. He lit a cigarette

Asher and Lascarides present (2.29) as another example of narration. This discourse relation is defined in terms that distinguishes it from, for example, continuation. Narration holds between (2.29a) and (2.29b), and between (2.29b) and $(2.29 \mathrm{c})$. These relations do not seem to be enthymematic - that someone enters a room is not a reason for that person sitting down, neither is sitting down an explanation for lighting a cigarette. There is no enthymematic argument in this discourse in need of a warrant. However, there is still some kind of expected progression in (2.29). We would probably have a harder time interpreting a discourse like $(2.30)$ as coherent:
(2.30) a. Max sat down
b. He came into the room
c. He lit a cigarette

Another problem arises when the content of a sentence is difficult to place in the same space and time as that of another sentence for world-knowledge reasons. For example, if $(2.29 \mathrm{~b})$ had been He dived into the Caribbean sea, rather than He sat down, the interpretation of the discourse as linked by narration would be less obvious. This indicates that some principles of world knowledge are underpinning narration, elaboration, continuation, etc. as well as explanation and result. We believe that this knowledge could be modelled in terms of topoi.

We fully agree with the perspective presented in SDRT in that a rhetorical element is necessary to fully capture the content of causal discourse relations, like explanation and result. Asher and Lascarides reject domain knowledge as a direct means of deciding which rhetorical relation we are dealing with in a particular discourse, since the principles which we draw on can often not be considered defaults which are true for the most part. For example, it is not the case that pushings normally result in fallings. However, we would like to argue that domain related resources in the form of topoi can supply an appropriate logical form to underpin a causal or consequential relation in discourse.

Discussing evidence for cognitive foundations for rhetorical relations, Asher and Lascarides refer to research according to which at least causal relations seem to help interpreters understand texts better (Flower and Hayes, 1980; Meyer and Freedle, 1984-referred to in Asher and Lascarides, 2003, p. 450). Since causal relations are what enthymemes are based on, this seems to sup- 
port the view that enthymemes help us structure discourse in a coherent way. Asher and Lascarides claim that the discourse relations in SDRT have semantic contents that relate to "fundamental conceptual categories by means of which we organise our beliefs", for example causation, sequencing, and part/whole. These categories could be seen as supertypes of more specific topoi, somewhat reminiscent of Aristotle's "common topoi" - topoi that may be used to underpin arguments within all domains.

\subsection{Summary}

In this chapter we have explored how enthymemes and topoi relate to a number of theories within pragmatics and philosophy of language. We noted that although pragmatic inferences are consistent with general principles like the maxim of relevance and assumptions of rationality and cooperation, these principles are not always enough to predict the actual inferences a language user makes in a given situation. Nor do they explain how a dialogue participant determines what contribution to make in order for an interlocutor to arrive at a particular conclusion. In response to this, we have sketched a picture of how enthymemes and topoi contribute to pragmatic processes by providing structure (enthymemes) and warrants (topoi) for reasoning.

We suggested that we could talk about accommodation of both topoi and enthymemes. Interpreting a discourse where the rhetorical structure is not explicit, a salient topos-one which by the language user is easily associated with the discourse situation - may be adopted as an underpinning for the discourse. Drawing on this topos the language user can accommodate an appropriate enthymeme, or some other type of rhetorical relation, which provides structure to the discourse. The discourse in (2.25) is an example of this.

Secondly, we may have an explicit enthymematic structure actually present in the discourse, as in Grice's example of a sentence giving rise to conventional implicature, (2.5a). In cases like this the structure already points to a topos, or at least at some instantiation of a topos, and the topos is thus more easily accommodated. However, in cases like this we can still expect the dialogue to be disrupted if we force a dialogue participant to accommodate a topos which is alien to him, or not salient in the situation.

We agreed with the anti-inferentialist view of cognitive resources underpinning pragmatic processes. However, we opposed the idea that these processes are either entirely associative, automatic and unconscious (as Recanati claims of primary pragmatic processes) or entirely inferential (as is claimed in Relevance Theory). Instead we argue that when dialogue participants make use 
of topoi which are established in the cognitive resources of the other dialogue participants, the pragmatic interpretations are more associative in nature, and when the dialogue participants are forced to accommodate novel or unexpected topoi, the process is mainly inferential. We suggested that topoi might be a good way of warranting not only enthymematic discourse relations, but also non-causal relations such as narration. 